\title{
The Effect Follicular Fluid Vitamin A, E, D and B6 on Embryo Morphokinetics and Pregnancy Rates in Patients Receiving Assisted Reproduction
}

\author{
Funda GODE ${ }^{1-2}$, Suleyman AKARSU ${ }^{3}$, Zeliha GUNNUR DIKMEN ${ }^{4}$, Burcu TAMER¹, Ahmet Zeki ISIK ${ }^{1}$ \\ Istanbul, Turkey
}

\section{ABSTRACT}

OBJECTIVE: To evaluate the associations among levels of vitamins A, E, D, and B6 in follicular fluid embryo morphokinetics and quality, and clinical pregnancy rates.

STUDY DESIGN: A total of 58 patients with unexplained infertility admitted to the in vitro fertilisation center of Izmir Medical Park Hospital were included in this prospective clinical study. For each patient, vitamin levels were assayed using high-performance liquid chromatography. After intracytoplasmic sperm injection, for each oocyte, the relationships between each vitamin and subsequent embryo quality, embryo morphokinetics, and clinical pregnancy rates were investigated. Embryos were classified as grade A, B, C, or D according to morphokinetic parameters using t5 - t2 and t5 - t3 (cc3).

RESULTS: There was no significant correlation between embryo morphokinetic parameters (tpnf, t2, $\mathrm{t} 3$, t4, t5, t6, t7 and t8) and follicular fluid vitamin (A, B6, D, and E) levels $(p>0.05)$. There was a significant positive correlation between t5 optimal and follicular fluid vitamin A levels $(p<0.05)$. There was a significant positive correlation between cc2 optimal and follicular fluid vitamin B6 levels $(p<0.05)$. Levels of vitamins $A$ and $B 6$ were significantly higher in grade $A$ and $B$ embryos than in grade $C$ and $D$ embryos. There were no significant relationships between vitamins $E$ or $D$ and embryo quality or between any vitamin and clinical pregnancy rates.

CONCLUSION: High levels of vitamins A and B6 in follicular fluid are significantly associated with highquality embryos and optimal morphokinetics. However, none of the vitamins considered showed a significant relationship with clinical pregnancy rates.

Keywords: Embryomorphokinetics, Vitamin A, Vitamin B6, Vitamin D, Vitamin E

Gynecol Obstet Reprod Med 2019;25(2):89-95

${ }^{1}$ Department of Obstetrics and Gynaecology, Bahcesehir University School of Medicine, Istanbul, Turkey

${ }^{2}$ Izmir Medical Park Hospital In Vitro Fertilization Unit, Izmir, Turkey

${ }^{3}$ Department of Obstetrics and Gynaecology, Yuksek Ihtisas University School of Medicine, Ankara, Turkey

${ }_{4}^{4}$ Department of Medical Laboratory Hacettepe University School of Medicine, Ankara, Turkey

Address of Correspondence: Funda Gode

Department of Obstetrics and

Gynaecology, Bahcesehir University

School of Medicine/ Istanbul

funda.gode@gmail.com

Submitted for Publication:

23.11.2018

Accepted for Publication:

14.02.2019

ORCID IDs of the authors:

F.G.: 0000-0002-9371-6942. S.A.: 0000-0001-6571-6403

Z.G.D.: 0000-0001-7154-090X. B.T.:0000-0001-8530-2128.

A.Z.I.: 0000-0002-4717-2665

\begin{tabular}{|c|c|}
\hline \multirow{3}{*}{ 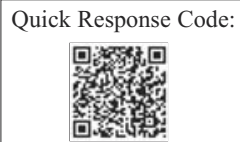 } & Access this article online \\
\hline & $\begin{array}{l}\text { Website: www.gorm.com.tr } \\
\text { e- mail: info@gorm.com.tr }\end{array}$ \\
\hline & DOI:10.21613/GORM.2018.860 \\
\hline
\end{tabular}

How to cite this article: Gode F. Akarsu S. Gunnur Dikmen Z. Tamer B. Isik AZ. Effect Follicular Fluid Vitamin A, E, D and B6 on Embryo Morphokinetics and Pregnancy Rates in Patients Receiving Assisted Reproduction. Gynecol Obstet Reprod Med 2019;25(2):89-95

\section{Introduction}

The microenvironment of the oocyte is an important factor in oocyte maturation; thus, the biochemical characteristics of the follicular fluid (FF) may play a critical role in oocyte quality, fertilisation, and subsequent embryonic development (1). The formation of reactive oxygen species (ROS) is a natural consequence of aerobic metabolism in oocytes, and is integral to maintaining oxygen homeostasis during oocyte maturation $(2,3)$. When the balance between oxidants and antioxidants is not maintained, the cellular environment becomes oxidatively stressed (3). ROS are associated with mitochondrial changes, meiotic arrest in oocytes, embryonic block, and cell death (4-7).

Vitamins and minerals are micronutrients that play important roles in many steps of antioxidant reactions. They may directly cause non-enzymatic antioxidant activity or may be required for enzymatic antioxidant activity $(4,8,9)$. Vitamins E and $\mathrm{A}$ are potent non-enzymatic antioxidants that protect the integrity of cell membranes and promote reproductive func- 
tion (10). Beta-carotene functions as an antioxidant in lipid phases by quenching singlet oxygen and scavenging peroxyl radicals $(11,12)$. Thus, vitamin A and carotenoids protect cells from superoxide radicals; in fact, low concentrations of these vitamins are correlated with anovulation $(10,13)$. Vitamin $E$ is a major chain-breaking antioxidant that destroys free radicals generated during ferrous ascorbate-induced lipid peroxidation $(4,14)$. Vitamin E protects mouse embryos against oxidative damage (15). In addition, in vitro administration of vitamin $\mathrm{E}$ improves the development of bovine embryos (16). Vitamin B6 is a coenzyme mainly involved in the metabolism of amino acids, nucleic acids, and lipids, and plays a role in the glutathione antioxidant defence system (17). It may also react directly with peroxy radicals, scavenge radicals, and inhibit lipid peroxidation $(17,18)$. Vitamin D in its biologically active form (1.25-dihydroxyvitamin D, or calcitriol) has antioxidant and anti-inflammatory effects in vitro (19-21). Some studies have suggested an impact of vitamin D on circulating oxidative and inflammatory markers $(21,22)$.

Treatment with additional micronutrients before in vitro fertilisation (IVF) cycles protect the follicular microenvironment against oxidative stress, thus increasing the number of good-quality oocytes (10). Higher intakes of antioxidants $\beta$-carotene, vitamin $C$, and vitamin $E$ are also associated with a shorter time to conception among couples undergoing treatment for unexplained infertility (23).

These findings suggest the importance of antioxidant vitamins in infertility patients. Although commercial supplements including these vitamins are commonly preferred in daily clinical practice in infertility clinics, interestingly, there is paucity of information on their exact roles in embryo quality and pregnancy rates. In addition, there are no data on the relationships between these vitamins and embryonic developmental kinetics. Therefore, we evaluated the associations among levels of vitamins $\mathrm{A}, \mathrm{E}, \mathrm{D}$, and $\mathrm{B} 6$ in FF, embryo morphokinetics and quality, and clinical pregnancy rates.

\section{Material and Method}

A total of 58 patients with unexplained infertility who underwent intracytoplasmic sperm injection (ICSI) cycles were included in this prospective cohort study. The study was approved by the Institutional Review Board (Approval number:1454). The study was conducted in accordance with the Declaration of Helsinki. Informed consent was obtained from all patients. The exclusion criteria were patient age $>41$ years old, severe oligoasthenoteratospermia, polycystic ovary syndrome, diminished ovarian reserve, endometriosis, the presence of hydrosalpinx, and any endocrinological disease. All patients underwent a gonadotropin-releasing hormone (GnRH) antagonist protocol for ovarian stimulation. Daily recombinant gonadotropin (150-300 IU, Gonal-F; Merck, Turkey) doses were started on the second menstrual day ac- cording to the patient's characteristics. When the leading follicle exceeded $14 \mathrm{~mm}$, a gonadotropin antagonist (Cetrotide, $0.25 \mathrm{mg}$; Merck) was started and human chorionic gonadotropin (hCG) (Ovitrelle, $250 \mu \mathrm{g}$; Merck) was administered when there were at least two follicles $>18 \mathrm{~mm}$. Transvaginal ultrasound-guided oocyte pick-up was performed $36 \mathrm{~h}$ after hCG injection.

Individual aspiration was applied for oocyte pick-up, and each follicle was recovered in a different tube. To avoid contamination from blood and flush medium or mixtures, only the FF from the first retrieved follicle, which contains a single oocyte-cumulus cell complex, was collected. After oocyte pick-up, oocytes isolated from FF samples were evaluated individually. One follicular sample per patient was used for analyses. FF samples were centrifuged at $2000 \mathrm{xg}$ for $10 \mathrm{~min}$ and the supernatants were stored at $-80^{\circ} \mathrm{C}$ until further analyses. Analyses of FF samples were performed only in patients with single-embryo transfer, for which an embryo was obtained from the oocyte of that follicle.

A time-lapse monitoring system (Embryoscope; Unisense FertiliTech, Denmark) in an atmosphere of 5\% $\mathrm{O}_{2}$ and $7 \%$ $\mathrm{CO}_{2}$ at $37^{\circ} \mathrm{C}$ was used to incubate embryos until transfer. Images of each embryo were taken every $20 \mathrm{~min}$ in seven different focal planes over $72 \mathrm{~h}$ of culture. Embryo Viewer software (Unisense Fertilitech Aarhus) was used to analyse embryonic images. The times from fertilisation to the following events were analysed: pronuclear fading (Pnf), when both pronuclei disappear; the first cleavage, when the zygote divides into two cells ( $\mathrm{t} 2$ ); and when cleavage giving rise to 3 to 9 cells were observed for the first time ( $\mathrm{t} 3, \mathrm{t} 4, \mathrm{t} 5, \mathrm{t} 6, \mathrm{t} 7, \mathrm{t} 8$, and t9, successively). The intervals between two consecutive cleavages were defined in the following manner: the duration of the second cell cycle $(\mathrm{cc} 2=\mathrm{t} 3-\mathrm{t} 2)$ was the time from the division into a two-blastomere embryo to the time of the division into a three-blastomere embryo, and second synchrony ( $\mathrm{s} 2=\mathrm{t} 4-\mathrm{t} 3)$ was the time from this division to a four-blastomere embryo. The duration of the third cell cycle ( $\operatorname{cc} 3=\mathrm{t} 5-\mathrm{t} 3)$ was the time from the division into a three-blastomere embryo to the time of division into a five-blastomere embryo. The embryos were graded according to the time-lapse parameters previously described by Basile et al. (24). The classification was based on the t5-t2 and cc3 intervals as follows: if the value of $\mathrm{t} 5$ - $\mathrm{t} 2$ was greater than $20.5 \mathrm{~h}$, the embryo was categorised as $\mathrm{A}$ or $\mathrm{B}$, and if the value fell outside the optimal range, the embryo was categorised as $\mathrm{C}$ or $\mathrm{D}$. If the value of cc3 was between 11 and $18 \mathrm{~h}$, the embryo was categorised as A or C, and if the value fell outside the optimal range, the embryo was categorised as B or D, depending on the $\mathrm{t} 5-\mathrm{t} 2$ value. Each FF analysis was performed for a single oocyte of a single embryo that was transferred to the patients. The relationship between vitamin A, E, D, and B6 content of each FF sample and subsequent embryo quality and morphokinetics were investigated. The relationships between vitamin levels optimal time- 
lapse parameters, including $\mathrm{t} 5, \mathrm{~s}^{2}$, and cc2, were also analysed, as were FF vitamin levels and pregnancy rates. Vitamin levels in each patient were determined by reversed-phase high-performance liquid chromatography (HPLC; Shimadzu Prominence, Japan).

Embryo transfer was performed on day 3 of development. For luteal support, all patients received $90 \mathrm{mg}$ progesterone gel (8\% Crinone gel, Merck) and $200 \mathrm{mg}$ vaginal micronised progesterone (Progestan caps, 200 mg; Kocak Farma, Turkey) daily. Biochemical pregnancy was determined when serum levels of $\beta$-hCG were $>20$ IU/I on Day 12 after embryo transfer, and clinical pregnancy was confirmed by ultrasound as the presence of foetal heart activity 8 weeks after embryo transfer.

\section{Statistical Analyses}

SPSS software (SPSS Inc., Chicago, IL, USA) was used for statistical analyses. Comparisons between two groups were performed using the independent t-test for parametric conditions and the Mann-Whitney U test for non-parametric conditions. Pearson and Spearman correlation analyses and receiver operating characteristic (ROC) curves were used. All values are expressed as the mean \pm standard deviation (SD). $P<0.05$ was considered to indicate statistical significance.

\section{Results}

The baseline characteristics of patients with high- and lowquality embryos according to embryo morphokinetic parameters are shown in table I. Group 1 consisted of patients with grades A and B (high-quality) embryo and group 2 consisted of patients with grades C and D (low-quality) embryos according to embryo morphokinetic parameters. There were no significant differences between groups according to age, body mass index (BMI), infertility duration, total gonadotropin dose, number of oocytes, number of MII oocytes, number of embryos, or serum levels of anti-Müllerian hormone (AMH), Day 3 follicle-stimulating hormone (FSH), luteinizing hor- mone (LH), estradiol, and vitamin D (Table I). Also there was not any correlation between baseline parameters and follicular fluid vitamin A, B6, E and D levels $(p>0.05)$.

Both normal (tpnf, t2, t3, t4, t5, t6, t7, and t8) and optimal (t5, s2, cc2) embryomorphokinetic parameters were evaluated. There was no significant correlation between normal embryomorphokinetic parameters (tpnf, t2, t3, t4, t5, t6, t7 and t8) and follicular fluid vitamin (A, B6, D, and E) levels $(p>0.05)$. There was a significant positive correlation between t5 optimal and follicular fluid vitamin A levels $(p<0.05)$. There was a significant negative correlation between s2optimal and follicular fluid vitamin D levels $(p<0.05)$. There was a significant negative correlation between cc2optimal and follicular fluid vitamin E levels $(p<0.05)$. There was a significant positive correlation between cc2 and follicular fluid vitamin B6 levels $(p<0.05)$. The embryos were graded according to optimal embryo morphokinetic parameters. When the relationship between follicular fluid vitamin levels and embryomorphokinetic grades were evaluated; vitamin A and B6 levels were significantly higher in grade $\mathrm{A}$ and $\mathrm{B}$ embryos than in grade $\mathrm{C}$ and $\mathrm{D}$ embryos (Table II). However, there were no significant correlations between vitamin E and D levels and embryo quality, or between any vitamin and clinical pregnancy rates (Table II).

Receiver operating characteristic curve analyses also indicated that higher embryo quality (grades A and B) was significantly associated with vitamin A and B6 levels (Figure 1). The area under the curve (AUC) for vitamin A was 70.4 (95\% confidence interval $[\mathrm{CI}], 0.536-0.872 ; p=0.025)$; that for vitamin B6 was 0.679 (95\% CI, 0.503-0.855; $p=0.050)$. Higher embryo quality was associated with vitamin D levels, but this difference was not significant. The AUC for vitamin D was 0.677 (95\% CI, 0.495-0.859; $p=0.052$ ). There was no association between embryo quality and vitamin E levels. The AUC for vitamin E was $0.586(95 \% \mathrm{CI}, 0.406-0.767 ; p=0.344)$. Finally, clinical pregnancy rates were not associated with vitamin levels.

Table I: Baseline characteristics of patients with high- and low-quality embryos according to embryo morphokinetic parameters

\begin{tabular}{lccc}
\hline & Group 1 & Group 2 & $p$ \\
\hline Age (years) & $31.15 \pm 4.56$ & $33.75 \pm 4.46$ & 0.066 \\
Infertility duration (months) & $54.41 \pm 37.15$ & $59.50 \pm 40.71$ & 0.710 \\
BMI $\left(\mathrm{kg} / \mathrm{m}^{2}\right)$ & $25.03 \pm 5.70$ & $28.83 \pm 5.68$ & 0.093 \\
$\mathrm{AMH}(\mathrm{ng} / \mathrm{mL})$ & $2.30 \pm 1.11$ & $1.91 \pm 1,20$ & 0.326 \\
Day3 FSH $(\mathrm{mLU} / \mathrm{mL})$ & $5.98 \pm 2.26$ & $5.35 \pm 2.05$ & 0.412 \\
E2 $(\mathrm{pg} / \mathrm{mL})$ & $43.42 \pm 47.71$ & $37.20 \pm 12.21$ & 0.661 \\
Total gonadotropin dose $(\mathrm{IU})$ & $2367.59 \pm 1011.82$ & $2647.91 \pm 778.43$ & 0.400 \\
Duration of induction (days) & $9.48 \pm 1.90$ & $9.08 \pm 1.24$ & 0.513 \\
Trigger Day E2 & $2122.77 \pm 1163.77$ & $1855.18 \pm 1441.87$ & 0.588 \\
Number of oocytes $(\mathrm{n})$ & $14.51 \pm 6.97$ & $8.91 \pm 4.62$ & 0.608 \\
Number of Mll oocytes $(\mathrm{n})$ & $11.96 \pm 6.08$ & $6.66 \pm 3.91$ & 0.131 \\
Number of embryos $(\mathrm{n})$ & $8.55 \pm 4.89$ & $21.13 \pm 10.01$ & 0.247 \\
Vit D (Plasma) $(\mu \mathrm{gg} / \mathrm{L})$ & $16.40 \pm 8.17$ & 0.200 \\
\hline
\end{tabular}

Group 1: Patients with grades $A$ and $B$ (high-quality) embryos according to embryo morphokinetic parameters.

Group 2: Patients with grades $C$ and $D$ (low-quality) embryos according to embryo morphokinetic parameters. 
Table II: Associations between levels of vitamins A, E, D, and B6 in follicular fluid and embryo quality and clinical pregnancy rates.

\begin{tabular}{|c|c|c|c|c|c|}
\hline \multirow{9}{*}{ Embryo quality } & Vitamin & & $\mathrm{n}$ & Mean \pm SD & $p$ \\
\hline & \multirow{2}{*}{ Vitamin A ( $\mu \mathrm{g} / \mathrm{L})$} & Grade A-B & 34 & $343.099 \pm 102.919$ & \multirow{2}{*}{$0.017^{*}$} \\
\hline & & Grade C-D & 24 & $263.716 \pm 100.653$ & \\
\hline & \multirow{2}{*}{ Vitamin E (mg/L) } & Grade A-B & 34 & $2.117 \pm 0.642$ & \multirow{2}{*}{0.505} \\
\hline & & Grade C-D & 24 & $2.452 \pm 2.691$ & \\
\hline & \multirow{2}{*}{ Vitamin $D(\mu g / L)$} & Grade A-B & 34 & $17.535 \pm 13.340$ & \multirow{2}{*}{0.999} \\
\hline & & Grade C-D & 24 & $17.537 \pm 29.144$ & \\
\hline & \multirow{2}{*}{ Vitamin B6 $(\mathrm{ng} / \mathrm{mL})$} & Grade A-B & 34 & $19.602 \pm 15.034$ & \multirow{2}{*}{$0.049^{*}$} \\
\hline & & Grade C-D & 24 & $12.521 \pm 9.927$ & \\
\hline \multirow{8}{*}{ Clinical Pregnancy } & \multirow{2}{*}{ Vitamin A $(\mu \mathrm{g} / \mathrm{L})$} & Non-pregnant & 26 & $309.398 \pm 120.441$ & \multirow{2}{*}{0.830} \\
\hline & & Pregnant & 32 & $315.438 \pm 92.879$ & \\
\hline & \multirow{2}{*}{ Vitamin E (mg/L) } & Non-pregnant & 26 & $2.249 \pm 2.059$ & \multirow{2}{*}{0.673} \\
\hline & & Pregnant & 32 & $2.086 \pm 0.652$ & \\
\hline & \multirow{2}{*}{ Vitamin $D(\mu \mathrm{g} / \mathrm{L})$} & Non-pregnant & 26 & $17.655 \pm 14.892$ & \multirow{2}{*}{0.971} \\
\hline & & Pregnant & 32 & $17.833 \pm 21.484$ & \\
\hline & \multirow{2}{*}{ Vitamin B6 $(\mathrm{ng} / \mathrm{mL})$} & Non-pregnant & 26 & $16.960 \pm 14.702$ & \multirow{2}{*}{0.685} \\
\hline & & Pregnant & 32 & $18.603 \pm 15.683$ & \\
\hline
\end{tabular}

$*: p<0.05$

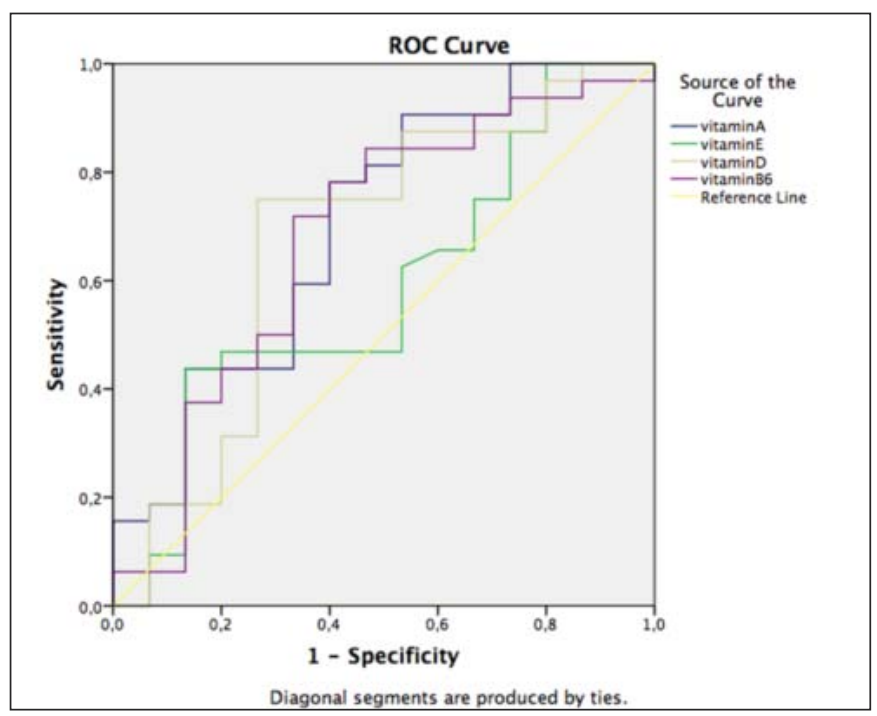

Figure 1: ROC curve analysis of vitamin $A, E, D$, and B6 levels for the prediction of embryo quality

\section{Discussion}

High FF vitamin A and B6 levels were significantly associated with high embryo quality and optimal morphokinetics. However, there were no significant associations between vitamin D and E levels and embryo quality, or between any vitamin and pregnancy rates. To the best of our knowledge, this is the first study to evaluate the relationships between levels of vitamins A, B6, E, and D in FF and embryo developmental kinetics. In addition, oocytes and embryos were continuously evaluated via embryoscope images, and embryo grading was performed according to morphokinetic parameters in an objective manner.

The role of vitamin A and its active metabolite retinoic acid during normal embryonic growth and development has been reported in previous animal studies. In addition to its antioxidant effects, vitamin $\mathrm{A}$ and its metabolites affect ovarian follicular growth, quality, and steroidogenesis (25-27). Currently, many gene products related to reproduction are modulated by vitamin A (28). It promotes cytoplasmic maturation in bovine oocytes via its modulatory effects on the gene expression of gonadotropin receptors (27). It also improves embryo quality after in vitro maturation of bovine and porcine oocytes $(25,26)$. However, there are very limited data on the relationship between vitamin A and human follicular development. Tiboni et al. reported that smokers have significantly lower levels of FF beta-carotene and lower fertilisation rates compared to non-smokers (29). In addition, FF vitamin A levels are associated with high-quality oocytes (30). Our results support these findings and the fundamental role of vitamin $\mathrm{A}$ in embryo quality.

We also found a positive correlation between FF vitamin B6 (pyridoxine) levels and embryo quality. The antioxidant properties of pyridoxine have recently been reported $(31,32)$. Because pyridoxine is essential for the metabolism of homocysteine into the amino acid cysteine, pyridoxine deficiency is correlated with elevated homocysteine levels and coronary artery disease (33). Vitamin B6 deficiency is also associated 
with problems in defence mechanisms against lipid peroxidation and exercise-induced oxidative stress $(34,35)$. Vitamin B6-deficient diets are correlated with increased plasma and tissue lipid peroxidation in rats (36). Despite these pertinent findings in animal studies, interestingly, there is no information regarding the relationship between vitamin B6 and embryonic development in humans in the literature. Our findings support a possible beneficial effect of vitamin B6 on oocyte and embryo quality.

Current data on vitamin D status and assisted reproductive technology outcomes are conflicting, but the majority of studies support vitamin D supplementation in deficient patients (37). Vitamin D deficiency is correlated with female infertility and poor IVF outcomes $(38,39)$. However, the exact mechanism of the positive effect of vitamin D is unclear. In a previous study, pregnancy rates were higher among women with high levels of vitamin D than among women with low levels, but there was no association between embryo quality and vitamin D status (40). The authors concluded that the effects of vitamin D on pregnancy rates may be related to the endometrium rather than oocytes. This hypothesis was supported by another study that reported lower pregnancy rates in vitamin D-deficient recipients of egg donation (41). In addition, in another study, FF vitamin D levels were not associated with oocyte quality and fertilisation, independent of pregnancy outcomes (42). Our study supports these reports, as we did not find any associations between vitamin D levels in FF and embryo quality or morphokinetic parameters.

We also did not find a relationship between vitamin E levels and embryo quality. Some animal studies have suggested that vitamin $\mathrm{E}$ improves in vitro maturation, fertilisation, and blastocyst development (43). However, some studies have not found any association between vitamin $\mathrm{E}$ and reproductive success [27,30]. In addition, in a previous study, the active form of vitamin E during oocyte maturation impaired the acquisition of oocyte developmental competence and also significantly diminished the percentage of blastocysts (44). The authors suggested that physiological concentrations of ROS may be required during oocyte maturation (44). Therefore, there may be upper and lower limits to the optimal antioxidant vitamin levels in FF for oocyte maturation.

Although dietary regulation or vitamin supplementation before IVF cycles is a very common management strategy, interestingly, there is a paucity of information on the subject. Therefore, we investigated the effects of FF levels of vitamins that are commonly found in multivitamin supplements. We did not find any associations between pregnancy rates and vitamin levels. However, the positive correlations between levels of vitamins A, D, and B6 and embryo quality suggest the importance of these vitamins to IVF outcomes. Thus, vitamin A and B6 supplementation before the start of IVF treatment cycles may be beneficial for improving embryo quality.
The limitations of our study were that we did not evaluate the plasma levels of these vitamins (except vitamin D), and did not evaluate the correlations between plasma and FF levels. Therefore, we cannot draw an absolute relationship between blood levels of these vitamins and embryo development. Another limitation of the study is small number of patients and larger number of studies are needed in this issue.

In conclusion, we found positive correlations between vitamin A and B6 concentrations in FF and subsequent embryo quality and morphokinetic parameters. Our results support the roles of vitamin A and B6 in early embryogenesis and embryo morphokinetics. Further research is necessary to elucidate the clinical effects of these vitamins on IVF outcomes.

:Acknowledgement: None

Conflict of Interest: None

Source of Funding: None

\section{References}

1. Revelli A, Piane LD, Casano S, Molinari E, Massobrio M, Rinaudo P. Follicular fluid content and oocyte quality: from single biochemical markers to metabolomics. Reprod Biol Endocrinol. 2009;7:40.

2. Castro L, Freeman BA. Reactive oxygen species in human health and disease. Nutrition. 2001;17(2):161-5.

3. Seifried HE, Anderson DE, Fisher EI, Milner JA. A review of the interaction among dietary antioxidants and reactive oxygen species. J Nutr Biochem. 2007;18(9):567-79.

4. Khazaei M, Aghaz F. Reactive oxygen species generation and use of antioxidants during in vitro maturation of oocytes. Int J Fertil Steril. 2017;11(2):63-70.

5. Kowaltowski AJ, Vercesi A. Mitochondrial damage induced by conditions of oxidative stress. Free Radic Biol Med 1999;26(3):463-71.

6. Nakamura Y, Yagamata Y, Sugino N, Takayama H, Kato H. Nitric oxide inhibits oocyte maturation. Biol Reprod. 2002;67(5):1588-92.

7. Hashimoto S, Minami N, Yamada M, Imai H. Excessice concentration of glucose during in vitro maturation impairs the developmental competence of bovine oocytes after in vitro fertilization: relevance to intracellular reactive oxygen species and glutathione contents. Mol Reprod Dev. 2000;56(4):520-6.

8. Pandey AN, Chaube SK. A moderate increase of hydrogen peroxide level is beneficial for spontaneous resumption of meiosis from diplotene arrest in rat oocytes cultured in vitro. Biores Open Acess. 2014;3(4):183-91.

9. Agarwal A, Gupta S, Sharma RK. Role of oxidative stress in female reproduction. Reprod Biol Endocrinol. 2005; $3: 28$.

10. Luddi A, Capaldo A, Focarelli R, Gori M, Morgante G, Piomboni $\mathrm{P}$, et al. Antioxidants reduce oxidative stress in 
follicular fluid of aged women undergoing IVF. Reprod Biol Endocrinol. 2016;14(1):57.

11. Ikeda S, Kitagawa M, Imai H, Yamada M. The roles of vitamin A for cytoplasmic maturation of bovine oocytes. J Reprod Dev. 2005;51(1):23-35.

12. Sies H, Stahl W. Vitamin E and C, beta-carotene, and other carotenoids as antioxidants. Am J Clin Nutr. 1995;62 (6 Suppl):1315-21.

13. Al-Azemi MK, Omu AE, Fatinikun T, Mannazhath N, Abraham S. Factors contributing to gender differences in serum retinol and alpha-tocopherol in infertile couples. Reprod Biomed Online. 2009;19(4):583-90.

14. Bansal AK, Bilaspuri GS. Antioxidant effect of vitamin E on motility, viability and lipid peroxidation of cattle spermatozoa under oxidative stress. Anim Sci Pap Rep. 2009;27(1):5-14.

15. Tsuji H, Muranaka M, Hamano K. Culture of in vitro mouse embryos with vitamin $\mathrm{E}$ improves development. J Reprod Dev. 2002;48:25-9.

16. Olson SE, Seidel GE Jr. Culture of in vitro-produced bovine embryos with vitamin $\mathrm{E}$ improves development in vitro and after transfer to recipients. Biol Reprod. 2000;62(2):248-52.

17. Hsu CC, Cheng CH, Hsu CL, Lee WJ, Huang SC, Huang YC. Role of vitamin B6 status on antioxidant defenses, gluthathione, and related enzyme activities in mice with homocysteine-induced oxidative stress. Food Nutr Res 2015;59:10:25702.

18. Keles M, Al B, Gumustekin K, Demircan B, Ozbey I, Akyuz M, et al. Antioxidative status and lipid peroxidation in kidney tissue of rats fed with vitamin B6- deficient diet. Ren Fail. 2010;32(5):618-22.

19. Alvarez JA, Zughaier SM, Law J, Hao L, Wasse H, Ziegler TR, et al. Effects of high-dose cholecalciferol on serum markers of inflammation and immunity in patients with early chronic kidney disease. Eur J Clin Nutr. 2013; 67(3):264-9.

20. Zhang Y, Leung DY, Richers BN, Liu Y, Remigio LK, Riches DW, et al. Vitamin D inhibits monocyte/ macrophage proinflammatory cytokine production by targeting MAPK phosphatase-1. J Immunol. 2012;188(5): 2127-35.

21. Alvarez JA, Chowdhury R, Jones DP, Martin GS, Brigham KL, Binongo JN, Ziegler TR, Tangpricha V. Vitamin D status is independently associated with plasma gluthatione and cysteine thiol/disulphide redox status in adults. Clin Endocrinol (Oxf). 2014;81(3):458-66.

22. Amer M, Qayyum R. Relation between serum 25-hydroxyvitamin $\mathrm{D}$ and C-reactive protein in asymptomatic adults (from continuous National Health and Nutrition Examination Survey 2001 to 2006) Am J Cardiol. 2012;109(2):226-30.

23. Ruder EH, Hartman TJ, Reindollar RH, Goldman MB.
Female dietary antioxidant intake and time to pregnancy among couples treated for unexplained infertility. Fertil Steril 2014;101(3):759-766.

24. Basile N, Nogales Mdel C, Bronet F, Florensa M, Riqueiros M, Rodrigo L, et al. Increasing the probability of selecting chromosomally normal embryos by timelapse morphokinetics analysis. Fertil Steril. 2014;101(3):699-704

25. Schweigert FJ, Zucker H. Concentrations of vitamin A, beta-carotene and vitamin $\mathrm{E}$ in individual bovine follicles of different quality. J Reprod Fertil. 1988;82(2):575-579.

26. Almiñana C, Gil MA, Cuello C, Caballero I, Roca J, Vazquez JM, et al. In vitro maturation of porcine oocytes with retinoids improves embryonic development. Reprod Fertil Dev. 2008;20(4):483-9.

27. Gómez E, Caamaño JN, Rodríguez A, De Frutos C, Facal N, Díez C. Bovine early embryonic development and vitamin A. Reprod Domest Anim. 2006;41(Suppl 2):63-71.

28. Schweigert FJ, Steinhagen B, Raila J, Siemann A, Peet D, Buscher U. Concentrations of carotenoids, retinol and $\alpha$ tocophero in plasma and follicular fluid of women undergoing IVF. Hum Reprod. 2003;18(6):1259-64.

29. Tiboni GM, Bucciarelli T, Giampietro F, Sulpizio M, Di llio C. Influence of cigarette smoking on vitamin E, vita$\min \mathrm{A}$, beta-carotene and lycopene concentrations in human pre-ovulatory follicular fluid. Int J Immunopathol Pharmacol. 2004;17(3):389-93.

30. Pauli SA, Session DR, Shang W, Easley K, Wieser F, Taylor R, et al. Analysis of follicular fluid retinoids in women undergoing in vitro fertilization: Retinoic Acid influences embryo quality and is reduced in women with endometriosis. Reprod Sci. 2013;20(9):1116-24.

31. Jain SK, Lim G. Pyridoxine and pyridoxamine inhibits superoxide radicals and prevent lipid peroxidation, protein glycosylation and $(\mathrm{Na}++\mathrm{K}+)$ ATPase activity reduction in high glucose treated human erythrocytes. Free Radic Biol Med. 2001;30(3):232-7.

32. Matxain JM. Theoretical study of the antioxidant properties of pyridoxine. J Phys Chem A. 2006;110(48); 1306872

33. Selhub J. Homocysteine metabolism. Annu Rev Nutr. 1999;19(1):217-46.

34. Choi EY, Cho YO. Effect of vitamin B6 deficiency on antioxidative status in rats with exercise-induced oxidative stress. Nutr Res Pract. 2009;3(3):208-11.

35. Ravichandran V, Selvam R. Increased lipid peroxidation in kidney of vitamin B6 deficient rats. Biochem Int. 1990;21(4):599-605.

36. Benderitter M, Hadj-Saad F, Lhuissier M, Maupoil V, Guilland JC, Rochette L. Effects of exhaustive exercise and vitamin B6 deficiency on free radical oxidative in male trained rats. Free Radic Biol Med. 1996;21(4):541-9. 
37. Pacis MM, Fortin CN, Zarek SM, Mumford SL, Segars JH. Vitamin D and assisted reproduction: should vitamin $\mathrm{D}$ be routinely screened and repleted prior to ART? A systematic review. J Assist Reprod Genet. 2015;32(3):32335.

38. Ozkan S, Jindal S, Greenseid K, Shu J, Zeitlian G, Hickmon C, et al. Replete vitamin D stores predict reproductive success following IV. Fertil Steril. 2010;94(4): 1314-9.

39. Garbedian K, Boggild M, Moody J, Liu KE. Effect of vitamin $\mathrm{D}$ status on clinical pregnancy rates following in vitro fertilization. CMAJ Open. 2013;1(2):E77-82.

40. Rudick B, Ingles S, Chung K, Stanczyk F, Paulson R, Bendikson K. Characterizing the influence of vitamin D levels on IVF outcomes. Hum Reprod. 2012;27(11): 33217.
41. Rudick BJ, Ingles SA, Chung K, Stanczyk FZ, Paulson $\mathrm{RJ}$, Bendikson KA. Influence of vitamin D levels on in vitro fertilization outcomes in donor-recipient cycles. Fertil Steril. 2014;101(2):447-52.

42. Farzadi L, Khayatzadeh Bidgoli H, Ghojazadeh M, Bahrami Z, Fattahi A, Latifi Z, et al. Correlation between follicular fluid 25-OH vitamin D and assisted reproductive outcomes. Iran J Reprod Med. 2015;13(6):361-6.

43. Tareq KMA, Akter QS, Khandoker MA, Tsuji H. Selenium and Vitamin E improve the in vitro maturation, fertilization and culture to blastocyst of porcine oocytes. J Reprod Dev. 2012;58(6):621-8.

44. Dalvit G, Llanes SP, Descalzo A, Insani M, Beconi M, Cetica P. Effect of alpha-tocopherol and ascorbic acid on bovine oocyte in vitro maturation. Reprod Dom Anim. 2005;40(2):93-7. 